Pacific

Journal of

Mathematics

\title{
SOME SHARP HARDY INEQUALITIES ON SPHERICALLY
} SYMMETRIC DOMAINS

FRANCESCO CHIACCHIO AND TONIA RICCIARDI 


\title{
SOME SHARP HARDY INEQUALITIES ON SPHERICALLY SYMMETRIC DOMAINS
}

\author{
FRANCESCO CHIACCHIO AND TONIA RICCIARDI
}

\begin{abstract}
We prove several sharp Hardy inequalities for domains with a spherical symmetry. In particular, we prove an inequality for domains of the unit $\boldsymbol{n}$ dimensional sphere with a point singularity, and an inequality for functions defined on the half-space $\mathbb{R}_{+}^{n+1}$ vanishing on the hyperplane $\left\{x_{n+1}=0\right\}$, with singularity along the $x_{n+1}$-axis. The proofs rely on a one-dimensional Hardy inequality involving a weight function related to the volume element on the sphere, as well as on symmetrization arguments. The one-dimensional inequality is derived in a general form.
\end{abstract}

\section{Introduction and main results}

Sharp Hardy inequalities have attracted a considerable attention in recent years, particularly in view of their applications to differential equations motivated by physics and geometry. Let $1<p<n$. The classical Hardy inequality states that

$$
\int_{\mathbb{R}^{n}}|D u|_{n}^{p} \geq\left(\frac{n-p}{p}\right)^{p} \int_{\mathbb{R}^{n}} \frac{|u|^{p}}{|x|_{n}^{p}}
$$

for all smooth functions $u$ compactly supported on $\mathbb{R}^{n}$, where $|x|_{n}^{2}=x_{1}^{2}+\cdots+x_{n}^{2}$ for all $x \in \mathbb{R}^{n}$. A considerable effort has been devoted to extending this inequality to manifolds, to special weight functions, and to domains exhibiting particular symmetries. See [Kufner and Persson 2003] for an extensive review. Our aim in this note is to derive some sharp Hardy type inequalities specifically tailored for manifolds with a spherical symmetry. Several recently proved inequalities concern the special case of the sphere; see [Bandle et al. 2005; Brezis and Peletier 2006]. Indeed, certain specific phenomena that do not occur on Euclidean space actually

MSC2000: primary 46E35; secondary 26D10, 35J25.

Keywords: sharp weighted Hardy inequalities, symmetrization.

Chiacchio is partially supported by GNAMPA-INdAM, Progetto "Proprietà analitico geometriche di soluzioni di equazioni ellittiche e paraboliche". Ricciardi is partially supported by Regione Campania L.R. 5/2006, by GNAMPA-INdAM, and by a PRIN-COFIN project of the Italian Ministry of University and Research.

Ricciardi is the corresponding author. 
do occur on spheres. For example, in [Bandle et al. 2005] it was shown that the Sobolev inequality admits minimizers on sufficiently large spherical caps.

To state our main results, we introduce some notation. Denote by

$$
\mathbb{S}^{n}=\left\{x=\left(x_{1}, \ldots, x_{n+1}\right) \in \mathbb{R}^{n+1}:|x|=1\right\}
$$

the unit $n$-sphere, where we set $|x|^{2}=|x|_{n+1}^{2}=x_{1}^{2}+\cdots+x_{n+1}^{2}$ for all $x \in \mathbb{R}^{n+1}$. For $1<p<n$ and for $a>0$, we define a weight function, which is related to the volume element on $\mathbb{S}^{n}$, by

$$
\tilde{\eta}_{a}:(0, a) \rightarrow \mathbb{R}, \quad t \mapsto \frac{(\sin t)^{-(n-1) /(p-1)}}{\int_{t}^{a}(\sin s)^{-(n-1) /(p-1)} d s} .
$$

Note that $\lim _{t \rightarrow 0^{+}} \tilde{\eta}_{a}(t)=\lim _{t \rightarrow a^{-}} \tilde{\eta}_{a}(t)=+\infty$; see (14) below. In Lemma 2.4 we will show that there exists a $\widetilde{T} \in(0, a)$ such that $\tilde{\eta}_{a}$ decreases in $(0, \widetilde{T})$ and increases in $(\widetilde{T}, a)$. Therefore, the truncated function

$$
\tilde{\eta}_{a, \tilde{T}}(t)= \begin{cases}\tilde{\eta}_{a}(t) & \text { if } t \in(0, \widetilde{T}), \\ \tilde{\eta}_{a}(\widetilde{T}) & \text { if } t \in[\widetilde{T}, a)\end{cases}
$$

is decreasing in $(0, a)$. We denote by $\Theta=\left(\theta_{1}, \ldots, \theta_{n-1}, \theta_{n}\right)$ the angular variables on $\mathbb{S}^{n}$ and to simplify notation we set $\theta=\theta_{n}$. The angle $\theta \in[0, \pi]$, satisfying $x_{n+1}=|x| \cos \theta$, will be the only relevant angular variable to our purposes. We denote by $g$ the standard metric on $\mathbb{S}^{n}$ and by $d V$ the volume element on $\mathbb{S}^{n}$. For $\alpha \in(0, \pi]$, we denote by $\mathscr{B}(\alpha)$ the geodesic ball (spherical cap) on $\mathbb{S}^{n}$ with radius $\alpha$ centered at the north pole $N=(0, \ldots, 0,1) \in \mathbb{R}^{n+1}$. Namely, we define

$$
\mathscr{B}(\alpha)=\left\{x \in \mathbb{S}^{n}: 0 \leq \theta<\alpha\right\} .
$$

Let $N \in \Omega \subset \mathbb{S}^{n}$ be an open set such that $|\Omega|<\left|\mathbb{S}^{n}\right|$, and let $a^{\star} \in(0, \pi)$ be such that $\left|\mathscr{B}\left(a^{\star}\right)\right|=|\Omega|$. Here $|E|$ denotes the volume of a measurable set $E \subset \mathbb{S}^{n}$ with respect to the standard Lebesgue measure induced by $g$ on $\mathbb{S}^{n}$. In turn, we define the weight function

$$
\rho_{a^{\star}}: \mathbb{S}^{n} \backslash\{N\} \rightarrow \mathbb{R}, \quad x \mapsto \begin{cases}\frac{p-1}{n-p} \tilde{\eta}_{a^{\star}, \tilde{T}}(\theta) & \text { if } x \in \mathscr{B}\left(a^{\star}\right) \backslash\{N\}, \\ \frac{p-1}{n-p} \tilde{\eta}_{a^{\star}, \tilde{T}}(\widetilde{T}) & \text { if } x \in \mathbb{S}^{n} \backslash \mathscr{B}\left(a^{\star}\right),\end{cases}
$$

where $\tilde{\eta}_{a^{\star}, \tilde{T}}$ is the weight function defined in (3) with $a=a^{\star}$.

Theorem 1.1. Let $n \geq 2$ and $1<p<n$. Let $\Omega \subset \mathbb{S}^{n}$ be an open set such that $N \in \Omega$ and $|\Omega|<\left|\mathbb{S}^{n}\right|$. Let $a^{\star}$ be such that $|\Omega|=\left|\Re\left(a^{\star}\right)\right|$. Then, for every $u \in W_{0}^{1, p}(\Omega)$,

$$
\int_{\Omega}|\nabla u|^{p} d V \geq\left(\frac{n-p}{p}\right)^{p} \int_{\Omega}|u|^{p} \rho_{a^{\star}}^{p} d V,
$$

and the constant $((n-p) / p)^{p}$ is sharp. 
Note that, since $\theta=d_{g}(x, N)$, we have

$$
\lim _{x \rightarrow N} d_{g}(x, N) \rho_{a^{\star}}(x)=1 ;
$$

see (27) below. Thus $\rho_{a^{\star}}$ is a natural extension of the classical singularity $|x|_{n}^{-p}$ appearing in (1).

Theorem 1.1, together with a Steiner symmetrization with respect to the angular variables, yields an inequality for functions defined on the half-space

$$
\mathbb{R}_{+}^{n+1}=\left\{x \in \mathbb{R}^{n+1}: x_{n+1}>0\right\}
$$

with singularity along the $x_{n+1}$-axis. More precisely, let $x^{\prime}=\left(x_{1}, \ldots, x_{n}, 0\right)$ for every $x \in \mathbb{R}^{n+1}$. We take $a^{\star}=\pi / 2$ in (2)-(4) and we define the singularity $\zeta$ as

$$
\zeta: \mathbb{R}_{+}^{n+1} \backslash\left\{x^{\prime}=0\right\} \rightarrow \mathbb{R}, \quad x \mapsto \rho_{\pi / 2}(x /|x|) .
$$

Note that $\zeta$ is singular on the $x_{n+1}$-axis.

Theorem 1.2. For every $u \in W_{0}^{1, p}\left(\mathbb{R}_{+}^{n+1}\right)$, we have

$$
\int_{\mathbb{R}_{+}^{n+1}}\left|D_{\Theta} u\right|^{p} d x \geq\left(\frac{n-p}{p}\right)^{p} \int_{\mathbb{R}_{+}^{n+1}}|u|^{p} \frac{\zeta^{p}(x)}{|x|^{p}} d x .
$$

Here $D_{\Theta} u(x)$ is the projection of the gradient $D u(x)$ on the sphere $\partial B(0,|x|)$. The constant $((n-p) / p)^{p}$ is sharp.

For special values of $p$ and $n$, the singularities appearing in (5) and (7) take particularly simple and explicit forms: Let $p=(n+1) / 2$ and suppose that $\Omega \subset \mathbb{S}^{n}$ is such that $|\Omega|=\left|\mathbb{S}^{n}\right| / 2$. Then, $a^{\star}=\pi / 2,(p-1) /(n-p)=1,(n-1) /(p-1)=2$ and therefore $\tilde{\eta}_{\pi / 2}(t)=\sin ^{-2} t\left(\int_{t}^{\pi / 2} \sin ^{-2} \sigma d \sigma\right)^{-1}=(\sin t \cos t)^{-1}$. Consequently, $\widetilde{T}=\pi / 4$ and

$$
\rho_{\pi / 2}(x)= \begin{cases}(\sin \theta \cos \theta)^{-1} & \text { if } x \in \mathscr{B}(\pi / 4) \backslash\{N\}, \\ 2 & \text { if } x \in \mathbb{S}^{n} \backslash \mathscr{B}(\pi / 4) .\end{cases}
$$

Note also that $(\sin \theta \cos \theta)^{-1}>\theta^{-1}$ for any $\theta \in(0, \pi / 4)$ and $2>\theta^{-1}$ for any $\theta \in(\pi / 4, \pi)$. Therefore, inequality (5) implies that

$$
\int_{\Omega}|\nabla u|^{p} d V \geq\left(\frac{n-p}{p}\right)^{p} \int_{\Omega}|u|^{p}\left(\frac{1}{d_{g}(x, N)^{p}}+h\right) d V,
$$

where $h$ is a positive quantity. This shows that (1) is improved on the sphere in this case. When $p=(n+1) / 2$ the same arguments also yield a simple form for (7). 
Indeed, in this case (7) may be written in the form

$$
\begin{aligned}
\int_{\mathbb{R}_{+}^{n+1}}\left|D_{\Theta} u\right|^{p} d x \geq\left(\frac{p-1}{p}\right)^{p}\left(\int_{\mathbb{R}_{+}^{n+1} \cap\{0 \leq \theta<\pi / 4\}}\right. & \frac{|u|^{p}}{\left|x^{\prime}\right|^{p}(\cos \theta)^{p}} d x \\
& \left.+2^{p} \int_{\mathbb{R}_{+}^{n+1} \cap\{\pi / 4 \leq \theta<\pi / 2\}} \frac{|u|^{p}}{|x|^{p}} d x\right) .
\end{aligned}
$$

This special case was also shown to be of interest in [Bandle et al. 2005].

An outline of our proofs is as follows. Our starting point is the one-dimensional Hardy inequality

$$
\int_{0}^{a}\left|u^{\prime}\right|^{p} \sin ^{n-1}(t) d t \geq\left(\frac{p-1}{p}\right)^{p} \int_{0}^{a}|u|^{p} \tilde{\eta}_{a, \tilde{T}}(t)^{p} \sin ^{n-1}(t) d t,
$$

for all $u$ such that $u(a)=0$, where $\tilde{\eta}_{a, \tilde{T}}$ is the function defined in (3). In fact, in Section 2 we shall prove some sharp weighted one-dimensional Hardy inequalities involving a general weight $\phi$; these reduce to (8) when $\phi(t)=\widetilde{\phi}(t)=\sin ^{n-1}(t)$; see Propositions 2.3 and 2.5 below. To this end, we extend a method described in [Hardy et al. 1952] — but see also [Tartar 1998] — for the special case $\phi(t)=1$. In fact, one of our efforts is to determine the general conditions on $\phi$ so that this method applies. This technique was also employed in [Brandolini et al. 2007] in the special case $\phi(t)=(2 \pi)^{-1 / 2} \exp \left\{-t^{2} / 2\right\}$ in the context of symmetrization with respect to Gaussian measure. On the other hand, our sharpness considerations as in Proposition 2.5 are new even in these special cases. In Section 3, we use spherical symmetrization to reduce Theorem 1.1 to (8). In turn, Theorem 1.1 together with a Steiner symmetrization with respect to the angular variables concludes the proof of Theorem 1.2.

\section{Some Hardy inequalities on intervals}

In this section we prove some weighted one-dimensional Hardy inequalities, as stated in Propositions 2.3 and 2.5. As already mentioned in Section 1, we will exploit a technique from [Hardy et al. 1952, Theorem 253, page 175]; see also [Brandolini et al. 2007; Tartar 1998]. Assume $a>0$ and $p>1$, and choose $\phi \in C^{1}((0, a]) \cap C^{0}([0, a])$ so that

$$
\phi(0)=0, \quad \phi(t)>0 \text { in }(0, a], \quad c_{1} t^{p-1+\delta} \leq \phi(t) \leq c_{2} t^{p-1+\delta},
$$

for some $c_{1}, c_{2}, \delta>0$. We let $W^{1, p}(0, a ; \phi)$ be the set of maps $u:[0, a] \rightarrow \mathbb{R}$ such that $u \in L_{\text {loc }}^{1}[0, a]$ and $\int_{0}^{a}\left|u^{\prime}\right|^{p} \phi d t<+\infty$, where $u^{\prime}$ denotes the distributional derivative of $u$. In it, we consider the subspace $\mathscr{E}=\left\{u \in W^{1, p}(0, a ; \phi): u(a)=0\right\}$, endowed with the norm $\|u\|=\left(\int_{0}^{a}\left|u^{\prime}\right| p \phi\right)^{1 / p}$. We note that if $u \in \mathscr{E}$, then $u$ is absolutely continuous in $[\epsilon, a]$ for all $\epsilon \in(0, a)$. On the other hand, $u$ is in general 
unbounded near the origin. Nevertheless, $u$ may be approximated in $\mathscr{E}$ by functions that vanish at 0 . More precisely we have the following.

Lemma 2.1. $C_{0}^{1}([0, a])$ is dense in $\mathscr{E}$.

Proof. Let $u \in \mathscr{E}$. By standard properties of Sobolev spaces, we may assume that $u \in C^{1}([\epsilon, a])$ for all $\epsilon \in(0, a)$. We first show that $C_{0}^{1}([0, a])$ is dense in $\mathscr{E} \cap L^{\infty}(0, a)$. Let $u \in \mathscr{E} \cap L^{\infty}(0, a)$. For $k \in \mathbb{N}$, we consider the sequence

$$
u_{k}(t)= \begin{cases}u\left(k^{-1}\right) k t & \text { if } t \in\left[0, k^{-1}\right], \\ u(t) & \text { if } t \in\left(k^{-1}, a\right] .\end{cases}
$$

By the elementary inequality $|\alpha+\beta|^{p} \leq 2^{p-1}\left(|\alpha|^{p}+|\beta|^{p}\right)$ for all $\alpha, \beta \in \mathbb{R}$,

$$
\begin{aligned}
\int_{0}^{a}\left|u^{\prime}-u_{k}^{\prime}\right|^{p} \phi d t & =\int_{0}^{k^{-1}}\left|u^{\prime}-u\left(k^{-1}\right) k\right|^{p} \phi d t \\
& \leq 2^{p-1}\left(\int_{0}^{k^{-1}}\left|u^{\prime}\right|^{p} \phi d t+\|u\|_{\infty}^{p} k^{p} \int_{0}^{k^{-1}} \phi d t\right) .
\end{aligned}
$$

Since $u^{\prime} \in L^{p}(0, a ; \phi)$, the absolute continuity of the Lebesgue integral implies that

$$
\int_{0}^{k^{-1}}\left|u^{\prime}\right|^{p} \phi d t=o(1) \quad \text { as } k \rightarrow+\infty .
$$

Then by (9), we have $\max _{t \in\left[0, k^{-1}\right]} \phi \leq c_{2} \max _{t \in\left[0, k^{-1}\right]} t^{p-1+\delta}=c_{2} k^{-p+1-\delta}$. Thus

$$
k^{p} \int_{0}^{k^{-1}} \phi d t \leq k^{p-1} \max _{t \in\left[0, k^{-1}\right]} \phi \leq c_{2} k^{-\delta}=o(1) \quad \text { as } k \rightarrow+\infty .
$$

From (10), (11) and (12), it follows that $\int_{0}^{a}\left|u^{\prime}-u_{k}^{\prime}\right|^{p} \phi d t=o(1)$ as $k \rightarrow+\infty$. We conclude by a standard regularization argument. Suppose that $u \in \mathscr{E}$. The sequence of bounded functions

$$
\hat{u}_{k}(t)= \begin{cases}u\left(k^{-1}\right) & \text { if } t \in\left[0, k^{-1}\right], \\ u(t) & \text { if } t \in\left(k^{-1}, a\right]\end{cases}
$$

satisfies

$$
\int_{0}^{a}\left|u^{\prime}-\hat{u}_{k}^{\prime}\right|^{p} \phi d t=\int_{0}^{k^{-1}}\left|u^{\prime}\right|^{p} \phi d t=o(1) \quad \text { as } k \rightarrow+\infty .
$$

We have reduced to the case where $u$ is bounded, and the claim is established.

Fix $a>0$. Let

$$
\eta_{a}(t)=\frac{\phi(t)^{-1 /(p-1)}}{\int_{t}^{a} \phi(\sigma)^{-1 /(p-1)} d \sigma} \quad \text { for } t \in(0, a) .
$$

We note that $\eta_{a}>0$ and furthermore the following holds. 
Lemma 2.2. The function $\eta_{a}$ defined by (13) satisfies

$$
\begin{aligned}
& \left(\frac{c_{1}}{c_{2}}\right)^{1 /(p-1)} \frac{\delta}{p-1} \frac{a^{\delta /(p-1)}}{t\left(a^{\delta /(p-1)}-t^{\delta /(p-1)}\right)} \\
& \quad \leq \eta_{a}(t) \leq\left(\frac{c_{2}}{c_{1}}\right)^{1 /(p-1)} \frac{\delta}{p-1} \frac{a^{\delta /(p-1)}}{t\left(a^{\delta /(p-1)}-t^{\delta /(p-1)}\right)}
\end{aligned}
$$

for all $t \in(0, a)$, where $c_{1}, c_{2}, \delta>0$ are the constants defined in (9).

Proof. We have

$$
\int_{t}^{a}\left(\sigma^{p-1+\delta}\right)^{-1 /(p-1)} d \sigma=\int_{t}^{a} \sigma^{-1-\delta /(p-1)} d \sigma=\frac{p-1}{\delta}\left(t^{-\delta /(p-1)}-a^{-\delta /(p-1)}\right) .
$$

Consequently,

$$
\frac{\left(t^{p-1+\delta}\right)^{-1 /(p-1)}}{\int_{t}^{a}\left(\sigma^{p-1+\delta}\right)^{-1 /(p-1)} d \sigma}=\frac{\delta}{p-1} \frac{a^{\delta /(p-1)}}{t\left(a^{\delta /(p-1)}-t^{\delta /(p-1)}\right)} .
$$

On the other hand, by the assumption (9) on $\phi$, we have

$$
\left(\frac{c_{1}}{c_{2}}\right)^{1 /(p-1)} \frac{\left(t^{p-1+\delta}\right)^{-1 /(p-1)}}{\int_{t}^{a}\left(\sigma^{p-1+\delta}\right)^{-1 /(p-1)} d \sigma} \leq \eta_{a}(t) \leq\left(\frac{c_{2}}{c_{1}}\right)^{1 /(p-1)} \frac{\left(t^{p-1+\delta}\right)^{-1 /(p-1)}}{\int_{t}^{a}\left(\sigma^{p-1+\delta}\right)^{-1 /(p-1)} d \sigma}
$$

and the asserted estimate follows.

For later use, we also note that $\eta_{a}$ satisfies a Riccati equation

$$
\eta_{a} \phi^{\prime} / \phi+(p-1) \eta_{a}^{\prime}=(p-1) \eta_{a}^{2} \text { in }(0, a) .
$$

The following Hardy inequality holds.

Proposition 2.3. Let $a>0$ and $p>1$, and suppose that $\phi$ satisfies (9). Let $\eta_{a}$ be correspondingly defined by (13). Then, for every $u \in \mathscr{E}$,

$$
\int_{0}^{a}\left|u^{\prime}\right|^{p} \phi d t \geq\left(\frac{p-1}{p}\right)^{p} \int_{0}^{a}|u|^{p} \eta_{a}^{p} \phi d t .
$$

The constant $((p-1) / p)^{p}$ is sharp.

Proof. In view of Lemma 2.1 we may assume that $u \in C_{0}^{1}((0, a))$. We recall the elementary convexity inequality $|\alpha|^{p} \geq|\beta|^{p}+p|\beta|^{p-2} \beta(\alpha-\beta)$ for all $\alpha, \beta \in \mathbb{R}$. Taking $\alpha=u^{\prime}$ and $\beta=-((p-1) / p) u \eta_{a}$, we derive

$$
\left|u^{\prime}\right|^{p} \geq\left|\frac{p-1}{p} u \eta_{a}\right|^{p}-p\left|\frac{p-1}{p} u \eta_{a}\right|^{p-2} \frac{p-1}{p} u \eta_{a}\left(u^{\prime}+\frac{p-1}{p} u \eta_{a}\right) .
$$


Multiplying by $\phi$ and integrating over $[0, a]$, we obtain

$$
\begin{aligned}
\int_{0}^{a}\left|u^{\prime}\right|^{p} \phi d t \geq\left(\frac{p-1}{p}\right)^{p}(1-p) \int_{0}^{a}|u|^{p} & \eta_{a}^{p} \phi d t \\
& -p\left(\frac{p-1}{p}\right)^{p-1} \int_{0}^{a}|u|^{p-2} u u^{\prime} \eta_{a}^{p-1} \phi d t .
\end{aligned}
$$

Integration by parts yields

$$
\begin{aligned}
\int_{0}^{a}\left|u^{\prime}\right|^{p} \phi d t \geq & \left(\frac{p-1}{p}\right)^{p}(1-p) \int_{0}^{a}|u|^{p} \eta_{a}^{p} \phi d t \\
& +\left(\frac{p-1}{p}\right)^{p-1} \int_{0}^{a}|u|^{p}\left(\eta_{a}^{p-1} \phi\right)^{\prime} d t-\left.\left(\frac{p-1}{p}\right)^{p-1}|u|^{p} \eta_{a}^{p-1} \phi\right|_{0} ^{a} .
\end{aligned}
$$

Now by (14) and the fact that $u \in C_{0}^{1}(([0, a]))$, we have $u \eta_{a} \in L^{\infty}(0, a)$. Therefore the boundary terms vanish and we obtain

$$
\begin{aligned}
& \int_{0}^{a}\left|u^{\prime}\right|^{p} \phi d t \\
& \geq(1-p)\left(\frac{p-1}{p}\right)^{p} \int_{0}^{a}|u|^{p} \eta_{a}^{p} \phi d t+\left(\frac{p-1}{p}\right)^{p-1} \int_{0}^{a}|u|^{p}\left(\eta_{a}^{p-1} \phi^{\prime}+(p-1) \eta_{a}^{p-2} \eta_{a}^{\prime} \phi\right) d t \\
& =(1-p)\left(\frac{p-1}{p}\right)^{p} \int_{0}^{a}|u|^{p} \eta_{a}^{p} \phi d t+\left(\frac{p-1}{p}\right)^{p-1} \int_{0}^{a}|u|^{p} \eta_{a}^{p-2}\left(\eta_{a} \frac{\phi^{\prime}}{\phi}+(p-1) \eta_{a}^{\prime}\right) \phi d t .
\end{aligned}
$$

In view of (15), we have

$$
\int_{0}^{a}|u|^{p} \eta_{a}^{p-2}\left(\eta_{a} \frac{\phi^{\prime}}{\phi}+(p-1) \eta_{a}^{\prime}\right) \phi d t=(p-1) \int_{0}^{a}|u|^{p} \eta_{a}^{p} \phi d t
$$

It follows that

$$
\begin{aligned}
\int_{0}^{a}\left|u^{\prime}\right|{ }^{p} \phi d t & \geq\left(\left(\frac{p-1}{p}\right)^{p}-\frac{(p-1)^{p}}{p^{p-1}}\right) \int_{0}^{a}|u|^{p} \eta_{a}^{p} \phi d t+\frac{(p-1)^{p}}{p^{p-1}} \int_{0}^{a}|u|^{p} \eta_{a}^{p} \phi d t \\
& =\left(\frac{p-1}{p}\right)^{p} \int_{0}^{a}|u|^{p} \eta_{a}^{p} \phi d t .
\end{aligned}
$$

Hence, (16) is satisfied.

Now we verify sharpness. Consider the sequence of functions $\left\{U_{k}\right\}_{k \in \mathbb{N}} \subset \mathscr{E}$ defined by

$$
U_{k}(t)= \begin{cases}\left(\int_{1 / k}^{a} \phi^{-1 /(p-1)} d \sigma\right)^{(p-1) / p} & \text { if } t \in[0,1 / k), \\ \left(\int_{t}^{a} \phi^{-1 /(p-1)} d \sigma\right)^{(p-1) / p} & \text { if } t \in[1 / k, a] .\end{cases}
$$


Then,

$$
U_{k}^{\prime}(t)= \begin{cases}0 & \text { if } t \in[0,1 / k), \\ -\frac{p-1}{p}\left(\int_{t}^{a} \phi^{-1 /(p-1)} d \sigma\right)^{-1 / p} \phi^{-1 /(p-1)}(t) & \text { if } t \in[1 / k, a] .\end{cases}
$$

We claim that

$$
\lim _{k \rightarrow+\infty} \frac{\int_{0}^{a}\left|U_{k}^{\prime}\right|^{p} \phi d t}{\int_{0}^{a} U_{k}^{p} \eta_{a}^{p} \phi d t}=\left(\frac{p-1}{p}\right)^{p} .
$$

Indeed, note that

$$
\left(\eta_{a}^{p} \phi\right)(t)=\frac{\phi^{-1 /(p-1)}(t)}{\left(\int_{t}^{a} \phi^{-1 /(p-1)} d \sigma\right)^{p}} \quad \text { for all } t \in(0, a) .
$$

Therefore, we may write

$$
\int_{0}^{a} U_{k}^{p} \eta_{a}^{p} \phi d t=A_{k}+B_{k}
$$

where

$$
A_{k} \equiv \int_{0}^{k^{-1}} U_{k}^{p} \eta_{a}^{p} \phi d t=\left(\int_{k^{-1}}^{a} \phi^{-1 /(p-1)} d \sigma\right)^{p-1} \int_{0}^{k^{-1}} \frac{\phi^{-1 /(p-1)}(t)}{\left(\int_{t}^{a} \phi^{-1 /(p-1)} d \sigma\right)^{p}} d t
$$

and

$$
\begin{aligned}
B_{k} \equiv \int_{k^{-1}}^{a} U_{k}^{p} \eta_{a}^{p} \phi d t & =\int_{k^{-1}}^{a}\left(\int_{t}^{a} \phi^{-1 /(p-1)} d \sigma\right)^{p-1} \frac{\phi^{-1 /(p-1)}(t)}{\left(\int_{t}^{a} \phi^{-1 /(p-1)} d \sigma\right)^{p}} d t \\
& =\int_{k^{-1}}^{a} \frac{\phi^{-1 /(p-1)}(t)}{\int_{t}^{a} \phi^{-1 /(p-1)} d \sigma} d t
\end{aligned}
$$

We claim that

$$
\lim _{k \rightarrow+\infty} A_{k}=1 /(p-1) .
$$

Indeed, we first observe that in view of (9), we have

$$
\int_{1 / k}^{a} \frac{\phi^{-1 /(p-1)}(t)}{\int_{t}^{a} \phi^{-1 /(p-1)} d \sigma} d t \geq\left(\frac{c_{2}}{c_{1}}\right)^{-1 /(p-1)} \int_{1 / k}^{a} \frac{t^{-1-\delta /(p-1)}}{\int_{t}^{a} \sigma^{-1-\delta /(p-1)} d \sigma} d t \rightarrow+\infty
$$

as $k \rightarrow \infty$. Hence, by l'Hôpital's rule, $\lim _{k \rightarrow+\infty} A_{k}$ is equal to the $k \rightarrow+\infty$ limit of either

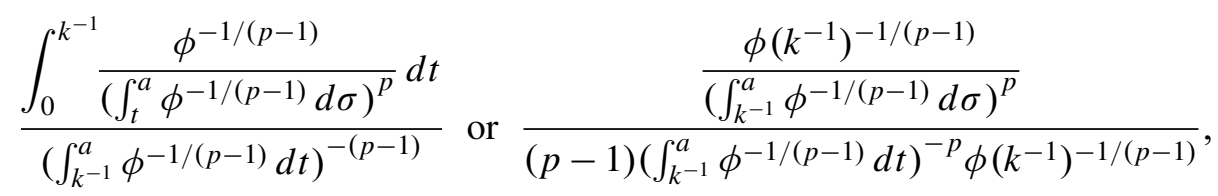


which then gives (21). We conclude that

$$
\int_{0}^{a} U_{k}^{p} \eta_{a}^{p} \phi d t=\int_{k^{-1}}^{a} \frac{\phi^{-1 /(p-1)}(t)}{\int_{t}^{a} \phi^{-1 /(p-1)} d \sigma} d t+\frac{1}{p-1}+o(1) .
$$

On the other hand, we have

$$
\int_{0}^{a}\left|U_{k}^{\prime}\right|^{p} \phi d t=\left(\frac{p-1}{p}\right)^{p} \int_{k^{-1}}^{a} \frac{\phi^{-1 /(p-1)}(t)}{\int_{t}^{a} \phi^{-1 /(p-1)} d \sigma} d t .
$$

Hence, recalling (22), we obtain

$\lim _{k \rightarrow+\infty} \frac{\int_{0}^{a}\left|U_{k}^{\prime}\right|^{p} \phi d t}{\int_{0}^{a} U_{k}^{p} \eta_{a}^{p} \phi d t}=\left(\frac{p-1}{p}\right)^{p} \lim _{k \rightarrow+\infty} \frac{\int_{k^{-1}}^{a} \frac{\phi^{-1 /(p-1)}}{\int_{t}^{a} \phi^{-1 /(p-1)} d \sigma} d t}{\int_{k^{-1}}^{a} \frac{\phi^{-1 /(p-1)}}{\int_{t}^{a} \phi^{-1 /(p-1)} d \sigma} d t+\frac{1}{p-1}+o(1)}$,

which is equal to $((p-1) / p)^{p}$. Hence, the sharpness is also established.

Now we show that under a simple extra assumption for $\phi$, the corresponding function $\eta_{a}$ defined by (13) has exactly one critical point, corresponding to the absolute minimum of $\eta_{a}$ in $(0, a)$.

Lemma 2.4. Suppose that $\phi:[0, a] \rightarrow \mathbb{R}$ satisfies (9). Also suppose that $\phi$ is twice differentiable in $(0, a)$ and that

$$
(\log \phi)^{\prime \prime}(t)=\left(\phi^{\prime} / \phi\right)^{\prime}(t)<0 \quad \text { for all } t \in(0, a) .
$$

Then there exists a unique $T \in(0, a)$ such that $\eta_{a}^{\prime}(t)<0$ in $(0, T)$ and $\eta_{a}^{\prime}(t)>0$ in $(T, a)$.

Proof. Differentiating the Riccati equation (15) we obtain

$$
\eta_{a}^{\prime} \phi^{\prime} / \phi+\eta_{a}\left(\phi^{\prime} / \phi\right)^{\prime}+(p-1) \eta_{a}^{\prime \prime}=2(p-1) \eta_{a} \eta_{a}^{\prime} .
$$

Suppose that $\eta_{a}^{\prime}(\hat{t})=0$. Then (24) implies that

$$
\eta_{a}^{\prime \prime}(\hat{t})=-\frac{1}{p-1} \eta_{a}(\hat{t})\left(\phi^{\prime} / \phi\right)^{\prime}(\hat{t})>0 .
$$

It follows that any critical point for $\eta_{a}$ is necessarily a strict minimum point. It then follows from Lemma 2.2 that $\eta_{a}$ admits a unique minimum point, and the existence of $T$ is established.

Let $\phi$ be twice differentiable and suppose that $\phi$ satisfies (9) and (23). Then, by truncating $\eta_{a}$ at the point $T$, we obtain the nonincreasing function

$$
\eta_{a, T}(t)= \begin{cases}\eta_{a}(t) & \text { for } t \in(0, T] \\ \eta_{a}(T) & \text { for } t \in(T, a]\end{cases}
$$


Since $\eta_{a, T} \leq \eta_{a}$ pointwise, it is clear that Proposition 2.3 still holds with $\eta_{a}$ replaced by $\eta_{a, T}$. On the other hand, it is not clear a priori whether, with such a replacement, the constant $((p-1) / p)^{p}$ is still sharp. This is indeed the case:

Proposition 2.5. Suppose that $\phi$ is twice differentiable and satisfies (9) and (23). Let $\eta_{a, T}$ be defined by (25). Then

$$
\int_{0}^{a}\left|u^{\prime}\right|^{p} \phi d t \geq\left(\frac{p-1}{p}\right)^{p} \int_{0}^{a}|u|^{p} \eta_{a, T}^{p} \phi d t \quad \text { for all } u \in \mathscr{E} .
$$

The constant $((p-1) / p)^{p}$ is sharp.

Proof. We need only check sharpness. For $k \in \mathbb{N}$ with $1 / k<T$, we consider the sequence $\left\{V_{k}\right\}_{k \in \mathbb{N}} \subset \mathscr{E}$ defined by

$$
V_{k}(t)= \begin{cases}U_{k}(t) & \text { if } t \in[0, T), \\ \frac{2 t-a-T}{T-a}\left(\int_{T}^{a} \phi^{-1 /(p-1)} d \sigma\right)^{(p-1) / p} & \text { if } t \in[T,(a+T) / 2), \\ 0 & \text { if } t \in[(a+T) / 2, a],\end{cases}
$$

where $\left\{U_{k}\right\}_{k \in \mathbb{N}}$ is the sequence defined in (18). Then

$$
V_{k}^{\prime}(t)= \begin{cases}U_{k}^{\prime}(t) & \text { if } t \in[0, T), \\ \frac{2}{T-a}\left(\int_{T}^{a} \phi^{-1 /(p-1)} d \sigma\right)^{(p-1) / p} & \text { if } t \in[T,(a+T) / 2), \\ 0 & \text { if } t \in[(a+T) / 2, a] .\end{cases}
$$

We claim that

$$
\lim _{k \rightarrow+\infty} \frac{\int_{0}^{a}\left|V_{k}^{\prime}\right|^{p} \phi d t}{\int_{0}^{a}\left|V_{k}\right|^{p} \eta_{a, T}^{p} \phi d t}=\left(\frac{p-1}{p}\right)^{p} .
$$

Let $C_{1}, C_{2}>0$ be defined by

$$
C_{1}=\left(\frac{p}{p-1}\right)^{p} \int_{T}^{(T+a) / 2}\left|V_{k}^{\prime}\right|^{p} \phi d t \quad \text { and } \quad C_{2}=\int_{T}^{(T+a) / 2}\left|V_{k}\right|^{p} \eta_{a, T}^{p} \phi d t .
$$

Note that $C_{1}$ and $C_{2}$ are independent of $k$. Then, we have

$$
\begin{aligned}
\frac{\int_{0}^{a}\left|V_{k}^{\prime}\right|^{p} \phi d t}{\int_{0}^{a} V_{k}^{p} \eta_{a, T}^{p} \phi d t} & =\left(\frac{p-1}{p}\right)^{p} \frac{\int_{k^{-1}}^{T} \frac{\phi^{-1 /(p-1)}}{\int_{t}^{a} \phi^{-1 /(p-1)} d \sigma} d t+C_{1}}{A_{k}+\int_{k^{-1}}^{T} \frac{\phi^{-1 /(p-1)}}{\int_{t}^{a} \phi^{-1 /(p-1)} d \sigma} d t+C_{2}} \\
& =\left(\frac{p-1}{p}\right)^{p} \frac{\int_{k^{-1}}^{T} \frac{\phi^{-1 /(p-1)}}{\frac{1}{p-1}+o(1)+\int_{k^{-1}}^{a} \frac{\phi^{-1 /(p-1)} d \sigma}{\int_{t}^{a} \phi^{-1 /(p-1)} d \sigma} d t+C_{1}}}{},
\end{aligned}
$$


where $A_{k}$ is defined in (19). This establishes the claim.

\section{Proofs of Theorems 1.1 and 1.2}

In this section we apply Proposition 2.5 to prove Theorems 1.1 and 1.2. In what follows, we assume that $1<p<n$. We let $a \in(0, \pi)$ and we take $\phi=\tilde{\phi}$, where $\tilde{\phi}(t)=\sin ^{n-1}(t)$. We note that $\tilde{\phi}$ satisfies assumptions (9) with $\delta=n-p$. The weight function corresponding to $\tilde{\phi}$ defined by (13) is given by (2), namely

$$
\tilde{\eta}_{a}(t)=\frac{(\sin t)^{-(n-1) /(p-1)}}{\int_{t}^{a}(\sin \sigma)^{-(n-1) /(p-1)} d \sigma} .
$$

Furthermore, $\tilde{\phi}$ is twice differentiable and we have

$$
(\log \tilde{\phi})^{\prime \prime}(t)=-\frac{n-1}{\sin ^{2} t} \quad \text { for all } t \in(0, \pi) .
$$

In particular, $\tilde{\phi}$ satisfies assumption (23). Using l'Hôpital's rule,

$$
\begin{aligned}
\frac{p-1}{n-p} \lim _{t \rightarrow 0^{+}} t \tilde{\eta}_{a, \tilde{T}}(t) & =\frac{p-1}{n-p} \lim _{t \rightarrow 0^{+}} \frac{t(\sin t)^{-(n-1) /(p-1)}}{\int_{t}^{a}(\sin \sigma)^{-(n-1) /(p-1)} d \sigma} \\
& =\frac{p-1}{n-p} \lim _{t \rightarrow 0^{+}} \frac{(1+o(1)) t^{-(n-p) /(p-1)}}{\int_{t}^{a} \sin \sigma^{-(n-1) /(p-1)} d \sigma}=1,
\end{aligned}
$$

and therefore (6) follows. The following elementary facts will be used in the sequel. Recall that for $x=\left(x_{1}, \ldots, x_{n}, x_{n+1}\right) \in \mathbb{R}^{n+1}$, we set $x_{n+1}=|x| \cos \theta$.

Lemma 3.1. Let $\Omega \subset \mathbb{S}^{n}$ and suppose that $u: \Omega \rightarrow \mathbb{R}$ depends on $\theta$ only. Then

$$
|\nabla u|^{2}=\left(\frac{\partial u}{\partial \theta}\right)^{2} \quad \text { and } \quad \int_{\mathscr{B}(a)} u(\theta) d V=\omega_{n-1} \int_{0}^{a} u(\theta) \tilde{\phi}(\theta) d \theta,
$$

where $\omega_{n-1}=(2 \pi)^{n / 2} / \Gamma(n / 2)$ denotes the volume of $\mathbb{S}^{n-1}$.

We shall also need some basic facts concerning spherical rearrangements; see for example [Baernstein and Taylor 1976; Sperner 1973]. For every $a \in[0, \pi]$, let

$$
A(a)=|\Re(a)|=\omega_{n-1} \int_{0}^{a} \tilde{\phi}(\theta) d \theta .
$$

Let $\Omega \subset \mathbb{S}^{n}$ be an open set, and let $u: \Omega \rightarrow \mathbb{R}$ be a measurable function. For $t>0$, let

$$
\mu(t)=|\{x \in \Omega:|u(x)|>t\}|
$$

denote the distribution function of $u$. Then the decreasing rearrangement $u^{*}$ of $u$ is defined in [Bennett and Sharpley 1988, page 39] by

$$
u^{*}(s)=\inf \{t \geq 0: \mu(t) \leq s\} \quad \text { for every } s \in[0,|\Omega|] .
$$


Let $\Omega^{\star}=\mathscr{B}\left(a^{\star}\right)$, where $a^{\star}=A^{-1}(|\Omega|)$. Then the spherical rearrangement $u^{\star}$ of $u$ is defined by $u^{\star}(x)=u^{*}(A(\theta))$ for $x \in \Omega^{\star}$. It follows that $u^{\star}$ is a decreasing function of $\theta$, and that its level sets are geodesic balls (spherical caps) centered at $N=(0,0, \ldots, 1) \in \mathbb{S}^{n}$. Since $|u|$ and $u^{\star}$ have the same distribution function, we have

$$
\int_{\Omega}|u|^{q} d V=\int_{\Omega^{\star}}\left(u^{\star}\right)^{q} d V \quad \text { for all } q \geq 1 .
$$

We shall use two standard inequalities involving rearrangements. The following lemma is a special case of the well-known Hardy-Littlewood inequality and may be found, for example, in [Bennett and Sharpley 1988, Theorem 2.2, page 44].

Lemma 3.2. Let $\Omega \subset \mathbb{S}^{n}$ be an open set and suppose that $u, v: \Omega \rightarrow \mathbb{R}$ are measurable and finite almost everywhere. Then

$$
\int_{\Omega} u v d V \leq \int_{\Omega^{\star}} u^{\star} v^{\star} d V
$$

The next inequality is a special case of the Pólya-Szegó principle, which may be found in [Aubin 1998, Proposition 2.17, page 41]; see also [Sperner 1973, theorem on page 325].

Lemma 3.3. Let $q \geq 1$ and let $u \in W^{1, q}\left(\mathbb{S}^{n}\right)$. Then

$$
\int_{\mathbb{S}^{n}}|\nabla u|^{q} d V \geq \int_{\mathbb{S}^{n}}\left|\nabla u^{\star}\right|^{q} d V
$$

Proof of Theorem 1.1. For every $u \in W_{0}^{1, p}(\Omega)$, by Lemmas 3.1 and 3.3, we have

$$
\int_{\Omega}|\nabla u|^{p} d V \geq \int_{\Omega^{\star}}\left|\nabla u^{\star}\right|^{p} d V=\omega_{n-1} \int_{0}^{a^{\star}}\left|\frac{\partial u^{\star}}{\partial \theta}\right|^{p} \tilde{\phi}(\theta) d \theta .
$$

On the other hand, in view of Lemma 3.2, we have

$$
\int_{\Omega}|u|^{p} \rho_{a^{\star}}^{p} d V \leq \int_{\Omega^{\star}}\left|u^{\star}\right|^{p} \rho_{a^{\star}}^{p} d V=\omega_{n-1} \int_{0}^{a^{\star}}\left|u^{\star}\right|^{p} \rho_{a^{\star}}^{p} \tilde{\phi}(\theta) d \theta .
$$

Therefore, it suffices to show that

$$
\int_{0}^{a^{\star}}\left|\frac{\partial u^{\star}}{\partial \theta}\right|^{p} \tilde{\phi}(\theta) d \theta-\left(\frac{n-p}{p}\right)^{p} \int_{0}^{a^{\star}}\left|u^{\star}\right|^{p} \rho_{a^{\star}}^{p} \tilde{\phi}(\theta) d \theta \geq 0 .
$$

This inequality holds by that definition of $\rho_{a^{\star}}$, as in (4), and by Proposition 2.5.

To show that the constant $((n-p) / p)^{p}$ is sharp, it suffices to use the sequence $\left\{\widetilde{V}_{k}\right\}_{k \in \mathbb{N}}$ of test functions obtained by setting $\phi=\tilde{\phi}, a=a^{\star}$ and $T=\widetilde{T}$ in (26). 
Namely,

(33) $\widetilde{V}_{k}(\theta)= \begin{cases}\left(\int_{k^{-1}}^{a^{\star}} \tilde{\phi}^{-1 /(p-1)} d \sigma\right)^{(p-1) / p} & \text { if } \theta \in\left[0, k^{-1}\right), \\ \left(\int_{t}^{a^{\star}} \tilde{\phi}^{-1 /(p-1)} d \sigma\right)^{(p-1) / p} & \text { if } \theta \in\left[k^{-1}, \widetilde{T}\right), \\ \frac{2 t-a^{\star}-\widetilde{T}}{\widetilde{T}-a^{\star}}\left(\int_{\widetilde{T}}^{a^{\star}} \tilde{\phi}^{-1 /(p-1)} d \sigma\right)^{(p-1) / p} & \text { if } \theta \in\left[\widetilde{T},\left(a^{\star}+\widetilde{T}\right) / 2\right), \\ 0 & \text { if } \theta \in\left[\left(a^{\star}+\widetilde{T}\right) / 2, a^{\star}\right] .\end{cases}$

This completes the proof of Theorem 1.1.

To prove Theorem 1.2, we use a Steiner-type symmetrization (see for example [Alvino et al. 1996; Kawohl 1985]) on $\mathbb{R}_{+}^{n+1}$ with respect to the angular variables. Let $u \in C_{0}^{1}\left(\mathbb{R}_{+}^{n+1}\right)$. For every fixed $r>0$, we consider the function obtained by restricting $u$ to $\mathbb{S}^{n} \cap \mathbb{R}_{+}^{n+1}$. Namely, we consider the function

$$
\Theta \in \mathbb{S}^{n} \cap \mathbb{R}_{+}^{n+1} \rightarrow u(r, \Theta),
$$

where $\Theta=\left(\theta_{1}, \ldots, \theta_{n-1}, \theta\right)$ is the set of all angular variables. We denote by $u^{*}(r, \cdot)$ the decreasing rearrangement of the function in (34), according to the definition given in (30). Finally we introduce the Steiner rearrangement $u^{\sharp}$ of $u$ by

$$
u^{\sharp}(r, \theta)=u^{*}(r, A(\theta)) \quad \text { for } \theta \in[0, \pi / 2],
$$

where $A(\theta)$ is defined in (29). We denote by $g_{r}$ the standard metric on $\mathbb{S}_{r}^{n}$ and by $d V_{r}$ the volume element on $\mathbb{S}_{r}^{n}$. Then, we have $D_{\Theta} u=\nabla_{g_{r}} u$ and therefore, in view of Lemma 3.1 and a rescaling argument,

$$
\left|D_{\Theta} u^{\sharp}(r, \theta)\right|^{p}=\frac{1}{r^{p}}\left|\frac{\partial u^{\sharp}}{\partial \theta}\right|^{p} .
$$

We claim that

$$
\frac{\zeta^{p}(x)}{|x|^{p}} \in L^{1}\left(\mathbb{R}_{+}^{n+1} \cap B_{R}\right) \quad \text { for every } R>0,
$$

where $\zeta$ is the weight function appearing in the statement of Theorem 1.2. Indeed, writing $x=\left(x^{\prime}, x_{n+1}\right)$, in view of (27), we have for some $C>0$

$$
\frac{\zeta(x)}{|x|} \leq \frac{C}{\theta r} \leq \frac{C}{r \sin \theta}=\frac{C}{\left|x^{\prime}\right|} .
$$

Consequently, for any $R>0$ we have

$$
\int_{\left|x^{\prime}\right|,\left|x_{n+1}\right|<R} \frac{\zeta^{p}(x)}{|x|^{p}} d x=\int_{0}^{R} d x_{n+1} \int_{\left|x^{\prime}\right|<R} \frac{\zeta^{p}(x)}{|x|^{p}} d x^{\prime} .
$$

Now (37) follows from the assumption $p<n$. 
Proof of Theorem 1.2. By density, it suffices to consider $u \in C_{0}^{1}\left(\mathbb{R}_{+}^{n+1}\right)$. If $\Omega \subset \mathbb{S}_{r}^{n}$ and $u: \Omega \rightarrow \mathbb{R}$ depends on $\theta$ only, then rescaling (28) gives

$$
\left|\nabla_{g_{r}} u\right|^{2}=\frac{1}{r^{2}}\left(\frac{\partial u}{\partial \theta}\right)^{2} \quad \text { and } \quad \int_{\mathscr{B}_{r}(\alpha)} u(\theta) d V_{r}=\omega_{n-1} r^{n} \int_{0}^{\alpha} u(\theta) \tilde{\phi}(\theta) d \theta,
$$

By Fubini's theorem and Lemma 3.3, we have

$$
\int_{\mathbb{R}_{+}^{n+1}}\left|D_{\Theta} u\right|^{p} d x=\int_{0}^{+\infty} \int_{\mathbb{S}_{r}^{n}}\left|\nabla_{g_{r}} u\right|^{p} d \sigma_{r} \geq \int_{0}^{+\infty} \int_{\mathbb{S}_{r}^{n}}\left|\nabla_{g_{r}} u^{\sharp}\right|^{p} d \sigma_{r},
$$

where $u^{\sharp}=u^{\sharp}(r, \theta)$ is defined in (35). Consequently, from (36), (39) and by Theorem 1.1 with $a^{\star}=\pi / 2$, we derive

$$
\begin{aligned}
\int_{\mathbb{R}_{+}^{n+1}}\left|D_{\Theta} u\right|^{p} d x & \geq \omega_{n-1} \int_{0}^{+\infty}\left(\int_{0}^{\pi / 2} \frac{1}{r^{p}}\left(\frac{\partial u^{\star}}{\partial \theta}\right)^{p} \sin ^{n-1} \theta d \theta\right) r^{n} d r \\
& \geq \omega_{n-1}\left(\frac{n-p}{p}\right)^{p} \int_{0}^{+\infty}\left(\frac{1}{r^{p}} \int_{0}^{\pi / 2}\left|u^{\star}\right|^{p} \zeta^{p} \sin ^{n-1} \theta d \theta\right) r^{n} d r \\
& \geq\left(\frac{n-p}{p}\right)^{p} \int_{\mathbb{R}_{+}^{n+1}} \frac{|u|^{p}}{r^{p}} \zeta^{p} d x .
\end{aligned}
$$

We are left to prove sharpness. We consider the sequence $u_{k}(\theta, r)=\Theta_{k}(\theta) R_{k}(r)$ for $k \in \mathbb{N}$, where $R_{k} \in C_{0}((0,+\infty))$ satisfies $R_{k}>0$ and $R_{k}^{p}(r) \stackrel{*}{\rightarrow} \delta_{1}(r)$, weakly in the sense of measures. Here $\delta_{1}$ denotes the Dirac mass on $(0,+\infty)$ centered at $r=1$, and $\Theta_{k}(\theta)=\widetilde{V}_{k}(\theta)$, where $\widetilde{V}_{k}$ is the sequence defined in (33), with $a^{\star}=\pi / 2$. We have

$$
\lim _{k \rightarrow+\infty} \int_{0}^{+\infty} R_{k}^{p}(r) r^{n} d r=\lim _{k \rightarrow+\infty} \int_{0}^{+\infty} R_{k}^{p}(r) r^{n-p} d r=1 .
$$

Now, the claim follows since

$$
\begin{aligned}
\frac{\int_{\mathbb{R}_{+}^{n+1}}\left|D_{\Theta} u_{k}\right|^{p} d x}{\int_{\mathbb{R}_{+}^{n+1}}\left|u_{k}\right| p \frac{\zeta^{p}}{r^{p}} d x} & =\frac{\int_{0}^{+\infty} R_{k}^{p}(r) r^{n} d r}{\int_{0}^{+\infty} R_{k}^{p}(r) r^{n-p} d r} \frac{\int_{\mathbb{S}_{r}^{n} \cap \mathbb{R}_{+}^{n+1}}\left|\Theta_{k}^{\prime}(\theta)\right|^{p} d v_{g_{r}} \cap \mathbb{R}_{+}^{n+1}\left|\Theta_{k}(\theta)\right|^{p} \zeta^{p} d v_{g_{r}}}{} \\
& =\frac{\int_{0}^{\pi / 2}\left|\Theta_{k}^{\prime}(\theta)\right|^{p} \tilde{\phi} d \theta}{\int_{0}^{\pi / 2}\left|\Theta_{k}(\theta)\right|^{p} \zeta^{p} \tilde{\phi} d \theta}+o(1)=\left(\frac{n-p}{p}\right)^{p}+o(1),
\end{aligned}
$$

where $o(1)$ vanishes as $k \rightarrow \infty$.

\section{References}

[Alvino et al. 1996] A. Alvino, G. Trombetti, J. I. Diaz, and P. L. Lions, "Elliptic equations and Steiner symmetrization”, Commun. Pure Applied Math. 49:3 (1996), 217-236. MR 96k:35036 Zbl 0856.35034 
[Aubin 1998] T. Aubin, Some nonlinear problems in Riemannian geometry, Springer, Berlin, 1998. MR 99i:58001 Zbl 0896.53003

[Baernstein and Taylor 1976] A. Baernstein, II and B. A. Taylor, "Spherical rearrangements, subharmonic functions, and *-functions in $n$-space", Duke Math. J. 43:2 (1976), 245-268. MR 53 \#5906 Zbl 0331.31002

[Bandle et al. 2005] C. Bandle, L. A. Peletier, and S. Stingelin, "Best Sobolev constants and quasilinear elliptic equations with critical growth on spheres", Math. Nachr. 278:12-13 (2005), 1388-1407. MR 2006h:35068 Zbl 02236553

[Bennett and Sharpley 1988] C. Bennett and R. Sharpley, Interpolation of operators, Pure and Applied Mathematics 129, Academic, Boston, MA, 1988. MR 89e:46001 Zbl 0647.46057

[Brandolini et al. 2007] B. Brandolini, F. Chiacchio, and C. Trombetti, "Hardy type inequalities and Gaussian measure", Commun. Pure Appl. Analysis 6:2 (2007), 411-428. MR 2008d:46044 Zbl 1154.46307

[Brezis and Peletier 2006] H. Brezis and L. A. Peletier, "Elliptic equations with critical exponent on spherical caps of $S^{3}$,, J. Anal. Math. 98 (2006), 279-316. MR 2007d:35077 Zbl 1151.35035

[Hardy et al. 1952] G. H. Hardy, J. E. Littlewood, and G. Pólya, Inequalities, 2nd ed., Cambridge University Press, 1952. MR 13,727e Zbl 0047.05302

[Kawohl 1985] B. Kawohl, Rearrangements and convexity of level sets in PDE, Lecture Notes in Mathematics 1150, Springer, Berlin, 1985. MR 87a:35001 Zbl 0593.35002

[Kufner and Persson 2003] A. Kufner and L.-E. Persson, Weighted inequalities of Hardy type, World Scientific, River Edge, NJ, 2003. MR 2004c:42034 Zbl 1065.26018

[Sperner 1973] E. Sperner, Jr., "Zur Symmetrisierung von Funktionen auf Sphären”, Math. Z. 134 (1973), 317-327. MR 49 \#5310 Zbl 0283.26015

[Tartar 1998] L. Tartar, "Imbedding theorems of Sobolev spaces into Lorentz spaces", Boll. Unione Mat. Ital. Sez. B Artic. Ric. Mat. (8) 1:3 (1998), 479-500. MR 99k:46060 Zbl 0929.46028

Received July 29, 2008.

FRANCESCO CHIACCHIO

Dipartimento di Matematica e Applicazioni "R. CACCioppoli"

UNIVERSITÀ DEGLI STUdi DI NAPOLI FEDERICO II

Via Cintia, Monte S. Angelo

80126 NAPOLI

ITALY

francesco.chiacchio@unina.it

TONIA RICCIARDI

Dipartimento di Matematica e Applicazioni "R. CACCioppoli"

UNIVERSITÀ DEGLI STUdI DI NAPOLI FEDERICO II

Via Cintia, Monte S. Angelo

80126 NAPOLI

ITALY

tonia.ricciardi@unina.it

http://wpage.unina.it/tonricci 